\title{
Correction to: Integrating Deep Offshore Wind with Pumped Hydro Storage in a Central Mediterranean Archipelago's Electricity Generation System
}

\author{
Robert N. Farrugia, Tonio Sant, and Cedric Caruana
}

\section{Correction to:}

Chapter 23 in: A. Sayigh (ed.), Mediterranean

Green Buildings \& Renewable Energy,

https://doi.org/10.1007/978-3-319-30746-6_23

The original version of the chapter was inadvertently published without incorporating the author' corrections to the acknowledgement section. The acknowledgement section has now been corrected. 